\title{
Emergências de saúde pública: conceito, caracterização, preparação e resposta
}

\author{
EDUARDO HAGE CARMO, GERSON PENNA \\ e WANDERSON KLEBER DE OLIVEIRA
}

\begin{abstract}
Aspectos conceituais
$\mathrm{N}$ AS ÚlTiMAS duas décadas e meia, o mundo vem sofrendo transformações que impactaram na saúde pública dos países, com reflexos na vida cotidiana das pessoas e na economia mundial. Nesse período, a poliomielite foi erradicada e as demais doenças imunopreveníveis tiveram uma intensa redução na sua ocorrência nas Américas e em grande parte dos países das demais regiões. Entretanto, cada vez mais vêm sendo registradas epidemias de doenças antigas como cólera, febre amarela e dengue, ao passo que novas doenças como Aids, febre hemorrágica pelo vírus ebola, hantavirose, febre do Nilo ocidental, síndrome respiratória aguda grave e influenza aviária passaram a afetar grandes contingentes populacionais ou vêm apresentando risco de disseminação. Essas epidemias estiveram relacionadas às importantes modificações ocorridas no mundo, com a intensificação do fluxo de pessoas, mercadorias e comércio entre os países.

Nesse mesmo período, o registro e a percepção de mudanças importantes no padrão de ocorrência das doenças infecciosas ou na dinâmica de transmissão dos seus agentes têm estimulado uma intensa reflexão sobre os fatores envolvidos nesse processo. Os sinais dessas mudanças têm sido evidenciados por meio de situações diversas, como o aparecimento de novos agentes infecciosos ou de modificações nos agentes já existentes, dotando-os de maior virulência e capacidade de gerar epidemias; aumento da capacidade de infectar diferentes espécies animais; desenvolvimento de resistência antimicrobiana; aumento na incidência de doenças infecciosas decorrentes de fatores conhecidos (migrações, mudanças climáticas, relaxamento em medidas de saúde pública) ou mesmo de fatores desconhecidos; introdução de doenças infecciosas em áreas indenes. Ademais, o desenvolvimento de novos métodos diagnósticos e os estudos clínicos e epidemiológicos vêm permitindo a detecção de agentes infecciosos até então desconhecidos ou mesmo o estabelecimento de vínculos etiológicos entre agentes e doenças já conhecidas (Barreto et al., 2006).

Buscando traduzir esse processo, o Instituto de Medicina dos Estados Unidos (Institute of Medicine, 1992) utilizou pela primeira vez o termo doenças
\end{abstract}


infecciosas emergentes, tendo sido modificado peloCenters for Disease and Control (CDC) dos Estados Unidos para "doenças de origem infecciosa cuja incidência em humanos tem aumentado dentro das últimas duas décadas ou ameaça aumentar no futuro próximo" (CDC, 1994). Esse conceito é impreciso, pois, ao caracterizar uma infecção emergente pelo aumento da sua ocorrência nas duas últimas décadas, estabelece um período de tempo artificialmente e não define qual o território em que o evento ocorre. Como o documento foi feito em torno das preocupações com o problema dessas infecções nos Estados Unidos, é evidente que o território oculto na definição refere-se ao desse país. Outro aspecto impreciso refere-se ao fato de que qualquer aumento na incidência de uma doença infecciosa seria caracterizado como emergência, independentemente da magnitude desse aumento e da tendência histórica anterior (Barreto et al., 2006).

Em 2003, o Instituto de Medicina atualizou o documento de 1992, modificando a definição anterior para "uma doença infecciosa clinicamente distinta, que tenha sido recentemente reconhecida, ou uma doença infecciosa conhecida cuja incidência esteja aumentando em um dado lugar ou entre uma população específica" (Institute of Medicine, 2003, p.32). Ainda que essa atualização torne o conceito mais preciso do ponto de vista espacial, persiste a imprecisão de que parâmetro de magnitude de incidência deva ser considerado para definir uma doença infecciosa como emergente.

A caracterização de uma doença infecciosa como emergente para ser mais precisa requer uma análise da ocorrência para cada doença de forma contextualizada para cada população, território e tempo histórico, nos quais as doenças incidem. Dessa forma, uma melhor utilização desse conceito deve considerar se a doença infecciosa e o seu surgimento ou o aumento na sua incidência têm relevância epidemiológica para uma população e território definido.

Com a aprovação do novo Regulamento Sanitário Internacional (RSI 2005 ) pela Assembléia Mundial da Saúde, em 2005 (WHA, 2005) ${ }^{1}$ um novo conceito foi estabelecido, desta vez não restrito às doenças de natureza infecciosa. O processo de revisão do RSI teve como importantes desafios estabelecer instrumentos mais adequados para o enfrentamento de problemas de saúde pública em escala mundial, que surgiram nas últimas décadas, e aperfeiçoar as medidas adotadas para os antigos problemas que já sofreram modificações ao longo do tempo, sem gerar maiores restrições ao comércio e às viagens internacionais (Fidler \& Gostin, 2006). Durante a revisão do RSI (2005), tendo como objetivo estabelecer medidas para ampliar as capacidades nacionais para detectar e responder aos riscos de disseminação de doenças entre os países, definiu-se como objeto de atenção desse Regulamento o que foi denominado de emergência de saúde pública de importância internacional.

O termo emergência de saúde pública de importância internacional é definido no RSI (2005), como (WHA, 2005, p.6):

Evento extraordinário, o qual é determinado, como estabelecido neste regulamento: 
- por constituir um risco de saúde pública para outro Estado² por meio da propagação internacional de doenças;

- por potencialmente requerer uma resposta internacional coordenada.

Ainda, segundo as definições do RSI (2005), "Evento significa a manifestação de uma doença ou uma ocorrência que cria um potencial para doença" (WHA, 2005, p.6). Portanto, os eventos não se limitam à ocorrência de dano (caso ou óbito por determinada doença), mas incluem fatores de risco para sua ocorrência. Também não são restritos à ocorrência de doenças transmissíveis, mas contemplam ainda problemas de saúde de natureza química, radionuclear ou decorrentes de desastres ambientais, como terremotos, inundações ou secas.

Dessa forma, o novo termo adotado no RSI (2005) pode tornar mais precisa e aplicável pelos sistemas nacionais de vigilância epidemiológica a definição de eventos que devem ser objeto de monitoramento, proposição e execução de ações que visem evitar ou diminuir a probabilidade de propagação internacional. Ademais, ao tratar de risco de disseminação de doenças, permite a adoção de medidas antecipatórias, com o potencial de evitar a ocorrência de dano à população. Com essas características, esse conceito se apresenta mais preciso que o conceito de doenças emergentes e mais amplo que o conceito de epidemia, na medida em que essas são restritas à ocorrência do dano. Além disso, algumas doenças emergentes e epidemias, sempre que representarem risco de disseminação internacional, podem se constituir em uma emergência de saúde pública de importância internacional.

Para que um evento seja considerado de importância internacional e, portanto, objeto do Regulamento, devem ser avaliadas algumas características próprias do evento e o contexto em que ocorre, que permitam classificá-lo como emergência de saúde pública de importância internacional. A forma em que essa avaliação é procedida baseia-se no uso do instrumento de decisão (Anexo 2 do RSI 2005) e avaliação de alguns critérios. Os eventos que deverão ser avaliados compreendem desde doenças específicas até aquelas em que a definição como entidade nosológica ainda não esteja estabelecida. Para as doenças específicas, serão adotadas duas formas de avaliação: para as doenças como varíola, poliomielite por vírus selvagem, influenza humana causada por novo subtipo de vírus e Sars, a notificação à Organização Mundial da Saúde (OMS) se dará de modo compulsório, independentemente da sua forma de ocorrência na população; para outras doenças que sob determinadas condições têm apresentado um maior risco de disseminação internacional, deve-se aplicar sempre o algoritmo de decisão quando da ocorrência de casos ou surtos, para avaliar se cumprem os critérios estabelecidos para notificação. Um evento ou uma doença específica deverá ser sempre notificado quando forem cumpridos alguns critérios avaliados de forma contextualizada para uma população, tempo e espaço específicos, além de considerar aspectos relacionados à probabilidade de restrições internacionais ao trânsito de pessoas, bens e mercadorias e ao comércio internacional. 
Para efeito da análise que será procedida no presente artigo, utilizaremos o conceito de emergência de saúde pública de importância nacional, derivado do que foi adotado no RSI (2005). Esse conceito que está sendo utilizado pela Secretaria de Vigilância em Saúde do Ministério da Saúde (SVS/MS) pode ser definido como: evento que apresente risco de propagação ou disseminação de doenças para mais de uma Unidade Federada (Estado ou Distrito Federal), com priorização das doenças de notificação imediata e outros eventos de saúde pública $^{3}$ (independentemente da natureza ou origem), depois de avaliação de risco, e que possa necessitar de resposta nacional imediata. De acordo com a Portaria de doenças e agravos de notificação imediata (Ministério da Saúde, 2006), considera-se um evento: cada caso suspeito ou confirmado de doença de notificação imediata; agregado de casos de doenças que apresentem padrão epidemiológico diferente do habitual (para doenças conhecidas); agregado de casos de doenças novas; epizootias e/ou mortes de animais que podem estar associadas à ocorrência de doenças em humanos (por exemplo, epizootia por febre amarela); outros eventos inusitados ou imprevistos, incluindo fatores de risco com potencial de propagação de doenças, como desastres ambientais, acidentes químicos ou radionucleares.

\section{Emergências de saúde pública no Brasil}

No presente tópico serão analisadas as emergências de saúde pública de importância nacional que foram notificadas à SVS/MS, a partir da implantação do Centro de Informações Estratégias e Respostas em Vigilância em Saúde (Cievs), desde março de 2006 até setembro de 2008. Esse Centro, que será descrito adiante, tem permitido o monitoramento contínuo de eventos que podem se constituir em emergências de importância nacional, após avaliação de risco, utilizando processo de análise semelhante ao adotado pelos países e pela OMS, com os instrumentos criados a partir da entrada em vigor do RSI (2005).

No período de estudos foram notificados ao Centro 543 eventos que poderiam representar emergências de saúde pública de importância nacional, com uma média de três eventos por semana epidemiológica. Cabe ressaltar que, de acordo com a definição de evento, conforme estabelecido na Portaria 05/2006 (Ministério da Saúde, 2006), cada caso de doença de notificação imediata representa uma unidade de análise; dessa forma, quando há uma agregação de casos relacionados epidemiologicamente, por exemplo, durante as epidemias, o número de eventos se torna superdimensionado.

O número de pessoas afetadas por possíveis emergências de saúde pública (exceto desastres) em todo o período foi de 281.060, e 765 evoluíram para óbito. Os desastres afetaram um número maior de pessoas (888.540), mas não necessariamente que sofreram danos à saúde. Dentre os eventos de natureza infecciosa, destaca-se a dengue que foi responsável pela ocorrência de 214.091 pessoas afetadas por evento em todo o período, seguido de influenza sazonal com 26.236 pessoas e doenças de transmissão alimentar, com 17.455 pessoas. 
$\mathrm{Na}$ distribuição dos eventos por Estado e município (Figura 1), observa-se que houve notificação de eventos em todos os Estados, com predomínio para os Estados de São Paulo (11\%), Minas Gerais (9\%), Rio Grande do Sul (9\%) e Pará $(8 \%)$. Entretanto, essa distribuição não é homogênea ao longo do período, relacionada seja com a melhoria da capacidade de detecção de cada estado, seja pela ocorrência de algumas emergências de saúde pública em alguns Estados em meses ou anos definidos.

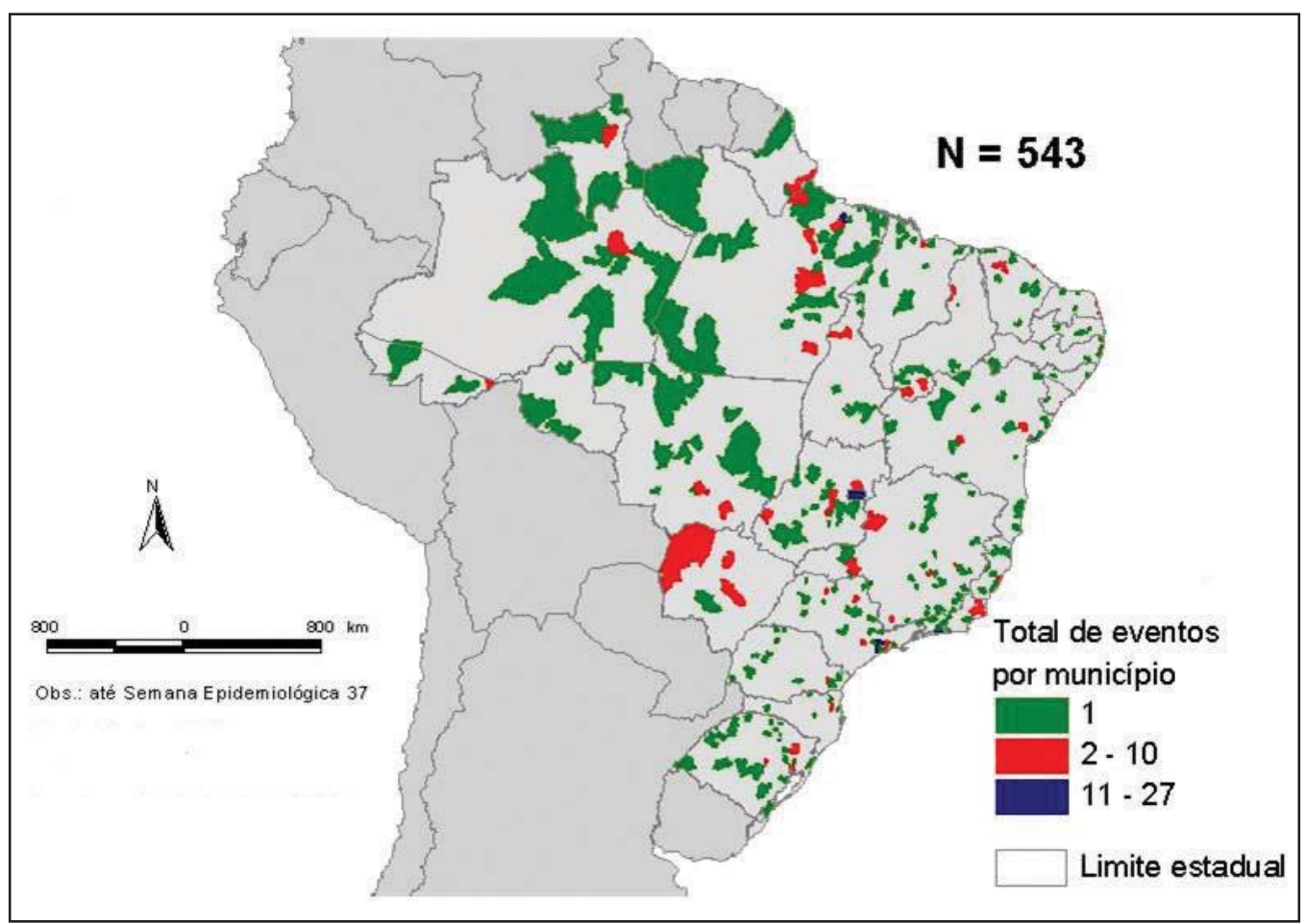

Fonte: MS/SVS/Cievs.

Figura l - Distribuição de possíveis emergências de saúde pública de importância nacional por município de referência. Brasil, março/2006 a setembro/2008.

Agrupando-se os eventos por modo de transmissão, similaridade do quadro clínico ou por natureza da exposição, observa-se um predomínio de antropozoonoses e doenças de transmissão hídrica ou alimentar, que representaram $29,1 \%$ e $22,5 \%$, respectivamente (Tabela 1). Dentre as antropozoonoses, $72 \%$ estavam relacionadas à ocorrência da emergência de saúde pública de febre amarela (nessa situação cada caso humano ou cada epizootia pela doença constituía um evento), ocorrida entre dezembro 2007 e maio de 2008, e aos surtos de doença de Chagas aguda, com predomínio de transmissão oral.

\section{Preparação e resposta às emergências de saúde pública}

Neste tópico serão destacadas as principais atividades desenvolvidas, no âmbito do Ministério da Saúde, que têm contribuído para o fortalecimento da 
capacidade nacional para detectar, prevenir e controlar emergências de saúde pública, no seu âmbito de atuação. Somadas às iniciativas estaduais e municipais, tem-se buscado dotar o país de um nível mais adequado de proteção à população para essas emergências.

Tabela l - Grupo de eventos notificados ao Cievs. De março/2006 a setembro/2008

\begin{tabular}{l|r|r|}
\multicolumn{1}{c|}{ Grupo de eventos (notificação) } & \multicolumn{1}{c|}{$\mathrm{n}$} & \multicolumn{1}{c}{$\%$} \\
\hline Zoonoses & 158 & 29,1 \\
Doenças de transmissão hídrica/alimentar & 122 & 22,5 \\
Doenças do sistema neurológico & 47 & 8,7 \\
Epizootias & 39 & 7,2 \\
Evento de etiologia indeterminada & 30 & 5,5 \\
Doença exantemática & 29 & 5,3 \\
Doenças transmitidas por vetores & 25 & 4,6 \\
Doenças do sistema respiratório & 23 & 4,2 \\
Evento adverso: produtos e procedimentos & 21 & 3,9 \\
Evento Inusitado & 15 & 2,8 \\
Infecção hospitalar & 13 & 2,4 \\
Intoxicação exógena & 13 & 2,4 \\
Desastre & 8 & 1,5 \\
\hline Total de eventos (até SE 37): & 543 & 100,0 \\
\hline
\end{tabular}

Fonte: MS/SVS/Cievs.

Implantação do Centro de Informações Estratégicas

e Respostas em Vigilância em Saúde (Cievs)

e da Rede Nacional de Alerta e Resposta às Emergências em Saúde Pública (Rede Cievs)

Desde março de 2006, encontra-se em funcionamento o Centro, que tem a função de detectar emergências de saúde pública, propiciar o gerenciamento dessas emergências e coordenar resposta apropriada pelo MS, junto com as secretarias estaduais e municipais de saúde. Constituído no âmbito da Secretaria de Vigilância em Saúde do Ministério da Saúde, o Centro opera continuamente ( 24 horas/dia, 365 dias/ano) e conta com estrutura tecnológica que possibilita ampliar a capacidade de uso de informações estratégicas e de comunicação com outras esferas de gestão do Sistema Único de Saúde (SUS). Esse Centro amplia a capacidade de detecção e resposta a toda emergência de saúde pública de importância nacional e pode ser uma ferramenta fundamental para o enfrentamento de pandemias, fortalecendo a capacidade de resposta já existente. Compõe 
uma rede mundial de alerta e resposta (Global Outbreak Alert and Response Network - Goarn), constituída por centros que têm a finalidade de detectar e apoiar a intervenção oportuna sobre emergências de saúde pública, visando evitar a propagação internacional de doenças. Além do Centro de Operações Estratégicas em Saúde (Strategic Health Operation Center - Shoc) existente na sede da OMS em Genebra - Suíça, há unidades em funcionamento em alguns países, como Estados Unidos, Canadá, México, Chile e Suécia, este pertencente à União Européia. O Brasil foi o terceiro país nas Américas a estruturar o seu centro de alerta e resposta.

Visando ampliar a capacidade de vigilância e resposta às emergências de saúde pública em todo o território nacional, tem havido investimento na implantação da Rede Cievs, composta por unidades de monitoramento e de equipes de respostas rápidas às emergências de saúde pública nos Estados e nas Secretarias de Saúde das capitais, constituindo assim a Rede Nacional de Alerta e Resposta às Emergências em Saúde Pública, denominada Rede Cievs. Essas unidades contam com estruturas similares ao Centro do Ministério da Saúde e estão adaptadas de acordo com a necessidade e capacidade de cada secretaria. Até o mês de outubro de 2008, a Rede Cievs já possuía vinte centros em estágio de implantação avançada ou em funcionamento e a meta é contar com centros implantados em todos os Estados e capitais até 2011.

O Centro utiliza informações procedentes de notificações realizadas por profissionais e serviços de saúde e secretarias municipais e estaduais. A notificação é realizada por e-mail institucional, telefone de acesso gratuito (0800) ou diretamente na página da SVS/MS (www.saude.gov.br/svs) em área específica para notificação de emergências. As fontes não-oficiais ("rumores") utilizadas são acessadas por meio de monitoramento diário de alguns veículos de comunicação (clipping), RSS feed (exemplo: Google Alert e similares), Promed, ou acesso às páginas de organizações internacionais como OMS, Centers for Disease Control and Prevention (CDC) dos Estados Unidos e de instituições de saúde de outros países.

A utilização de novos indicadores de monitoramento de emergências de saúde pública pelo Centro tem permitido avaliar a oportunidade do Sistema de Vigilância em Saúde para detectar e responder às emergências. Assim, foram criados os seguintes indicadores: oportunidade de notificação - tempo (em dias) entre a data do início do evento ou do início dos sintomas do(s) primeiro(s) caso(s) e data de recebimento da notificação ao Centro; oportunidade de encerramento - tempo (em dias) entre a data da notificação ao Centro e a data do encerramento do evento. Na avaliação desses indicadores, a mediana de oportunidade de notificação no período de análise foi de dez dias, enquanto a mediana de oportunidade de encerramento foi de quinze dias. A continuidade da avaliação desses indicadores anualmente, bem como a sua validação e aperfeiçoamento, permitirá avaliar o desempenho da Rede Cievs e dos demais componentes do Sistema de Vigilância em Saúde para detectar e responder às emergências. 


\section{Formação de técnicos para investigação e resposta às emergências de saúde pública}

Desde o ano de 2000, vem sendo desenvolvido pelo Ministério da Saúde o Programa de Treinamento em Epidemiologia Aplicada aos Serviços do SUS, em colaboração com os CDC , contando atualmente com 59 profissionais já formados e dezenove em treinamento. Esse treinamento em serviço, realizado por um período de dois anos no âmbito da SVS/MS, habilita profissionais de saúde pública em detecção, investigação, análise e resposta às emergências de saúde pública, com atividades em campo e atividades teóricas. Enquanto nos primeiros anos o Episus era voltado para formação de técnicos que iriam compor as equipes da SVS/MS do Ministério da Saúde, atualmente muitos técnicos formados compõem as equipes de outros órgãos do SUS (Agência Nacional de Vigilância Sanitária, secretarias estaduais e municipais de Saúde).

Nos últimos anos, tem sido apoiada a descentralização desse treinamento para as secretarias estaduais de Saúde, acompanhando a formação de unidades de respostas rápidas nos Estados, com o objetivo de que todos os Estados contem com profissionais adequadamente capacitados para detectar, investigar e responder às emergências de saúde pública no seu âmbito de atuação.

Atualmente, o Episus compõe a Rede Cievs, agregando, dessa forma, a estrutura e os instrumentos necessários para detectar e monitorar as emergências com os profissionais treinados em investigação epidemiológica e resposta a essas emergências.

Desde a implantação do Episus, os profissionais foram responsáveis, em conjunto com outros técnicos das áreas do Ministério da Saúde, SES e SMS, pela investigação epidemiológica de 157 emergências de saúde pública, contribuindo para a elucidação desses eventos e sua resolução, incluindo cooperações internacionais, como a realizada em 2008 durante investigação de surto de cólera, em Guiné-Bissau.

Para o aperfeiçoamento da Rede Cievs, o Ministério da Saúde está estruturando um curso de especialização em monitoramento de emergências e epidemiologia de campo, voltado para a preparação e resposta às emergências em saúde pública, além de uma nova formatação para o Curso Básico de Vigilância em Saúde, incorporando ferramentas de ensino a distância e componentes de vigilância ambiental e sanitária. Dessa forma, incluindo o Episus, a Rede Cievs terá à disposição três estratégias complementares de capacitação dos profissionais para o enfrentamento de emergências em saúde pública.

\section{Implantação de rede de vigilância epidemiológica hospitalar}

Visando à detecção precoce de doenças graves com potencial de disseminação, que são detectadas pela rede hospitalar, bem como promover uma maior integração com esta fonte de notificação para a vigilância epidemiológica, vêm sendo realizados investimentos em hospitais em todos os Estados, para implantação e fortalecimento de núcleos de vigilância epidemiológica hospitalar. Desde 
a sua implantação, a partir de 2005 até outubro de 2008, foram implantados ou apoiados 192 núcleos de vigilância epidemiológica hospitalar.

\section{Ampliação da capacidade laboratorial}

Nos últimos anos foi constituída e ampliada a rede de laboratórios de saúde pública, que conta atualmente com laboratórios para detecção das principais doenças com potencial de disseminação em todos os Estados e no Distrito Federal, três laboratórios de referência nacional e internacional e quatorze laboratórios de fronteira. A rede conta com financiamento e fornecimento de insumos estratégicos para diagnóstico das principais doenças sujeitas à vigilância epidemiológica, assim como para detecção de emergências de saúde pública, providos pelo Ministério da Saúde e pelas secretarias estaduais. Como atividade estratégica para prover a rede de métodos modernos e mais oportunos para diagnóstico laboratorial, têm sido realizados investimentos com a meta de implantar biologia molecular em todos os Laboratórios Centrais dos Estados. Visando ainda assegurar condições adequadas de biossegurança para a realização de exames em laboratórios estratégicos da rede, foram estabelecidas normas de biossegurança e realizados investimentos para dotar doze laboratórios com nível de segurança 3.

\section{Preparação para pandemia de influenza}

Tendo em vista a possibilidade da ocorrência de uma pandemia de influenza por um novo subtipo do virus, o Ministério da Saúde, em colaboração com outros órgãos do governo federal, vem estruturando a rede de serviços do SUS para dar uma resposta adequada a essa eventual pandemia. Dentre as medidas implementadas destacam-se: a elaboração e atualização do Plano Nacional e dos planos estaduais de Preparação; a constituição de um Grupo Executivo Interministerial, que tem sido responsável pelo gerenciamento do Plano e acompanhamento das ações desenvolvidas pelos diversos órgãos do governo federal; a aquisição e produção de insumos - com aquisição de antivirais para uso durante uma eventual pandemia e desenvolvimento da capacidade de produção nacional de vacinas, por meio do Instituto Butantã, tanto para uso durante as campanhas nacionais contra influenza "sazonal" quanto para uso em uma situação de pandemia; a aquisição de insumos e capacitação da rede nacional de laboratório de saúde pública para detecção dos vírus influenza, incluindo a cepa potencialmente pandêmica; o monitoramento de vírus influenza em aves migratórias, por meio da realização de inquéritos sorológicos em áreas de pouso e invernada, em parceria com o Ministério da Agricultura, Pecuária e Abastecimento; o estabelecimento da notificação imediata de caso ou surto de influenza humana por novo subtipo; a elaboração de planos de preparação específicos nas áreas de pontos de entrada (portos e aeroportos), agricultura, comunicação, logística, defesa e defesa civil.

\section{Implementação do Regulamento Sanitário Internacional (2005)}

Dentre as atividades iniciais para implementação do RSI (2005) no país e visando coordenar esse processo, o Ministério da Saúde designou a SVS/MS 
como Ponto Focal Nacional. Com o objetivo de contar com permanente apoio em atividades estratégicas para a implementação do RSI (2005) no país, a SVS/ MS designou o Instituto de Saúde Coletiva (ISC/UFBA) como Centro Colaborador. Dentre as atividades que já vêm sendo desenvolvidas por esse Instituto, destacam-se o aperfeiçoamento do instrumento de decisão para classificação das emergências de saúde pública e a avaliação de capacidades básicas de vigilância e resposta às emergências de saúde pública, relacionadas adiante.

Desde 2002, o Ministério da Saúde vem avaliando e contribuindo para o aperfeiçoamento do instrumento de decisão para classificação de eventos que podem constituir uma Emergência de Saúde Pública de Importância Internacional, com base em análise dos relatórios das investigações das emergências epidemiológicas que ocorreram no país. A partir de 2006, a SVS/MS vem colaborando com a OMS na avaliação desse instrumento de decisão e no desenvolvimento de um instrutivo para sua aplicação. Esse instrutivo possibilitará que o instrumento de decisão seja utilizado de forma mais precisa e homogênea por todos os países e, no interior desses, por todos os Estados.

Desde o ano de 2000, o Brasil vem desenvolvendo ações coordenadas com os países do Mercosul e demais países sul-americanos, inicialmente durante o processo de revisão do RSI e, a partir de 2005, visando apoiar a implementação do RSI (2005) na região. Dentre as atividades estratégicas desenvolvidas, destaca-se a avaliação de capacidades básicas de vigilância e resposta às emergências de saúde pública (Anexo 1 do RSI 2005). Os países do Mercosul desenvolveram um instrumento para avaliação das capacidades básicas de vigilância epidemiológica e pontos de entrada internacional (portos, aeroportos e fronteiras) e vêm contribuindo com a Opas/OMS para que esteja disponível para utilização por outros países das Américas; dessa forma, essa avaliação poderá ser realizada de forma mais apropriada para a realidade dos serviços de saúde dos países da região.

Com a disponibilidade desse instrumento, a SVS/MS, com a colaboração do ISC/UFBA, iniciou a avaliação das capacidades, com o objetivo de identificar a situação dos serviços de vigilância epidemiológica, laboratório de saúde pública e rede de atenção para detecção e resposta às emergências de saúde pública, apontar as necessidades de aperfeiçoamento e identificar prioridades para investimento futuro. Essa atividade será desenvolvida até junho de 2009 e compreenderá os três níveis de atenção da rede de vigilância e resposta. Outro instrumento também elaborado pelos países do Mercosul para avaliar as capacidades básicas em pontos de entrada internacional vem sendo aplicado pela Anvisa nos portos, aeroportos e pontos de fronteira definidos por essa Agência, de acordo com critérios epidemiológicos e de fluxos de pessoas, bens e mercadorias.

Como próximo desafio, destaca-se a estruturação do Plano Nacional de Preparação e Resposta às Emergências em Saúde Pública, conforme requerido pelo RSI. Esse plano irá agregar todas as iniciativas e planos isolados, buscando identificar as ações comuns e o fortalecimento das específicas, buscando otimizar 
recursos e melhorar a oportunidade das ações para resposta apropriada de saúde pública.

\section{Discussão}

Os novos cenários epidemiológicos representados pelo surgimento de novas doenças transmissíveis, modificações nos padrões habituais de transmissão de doenças existentes, ampliação da importância das doenças e dos agravos nãotransmissíveis na agenda de saúde pública, o surgimento e a propagação de doenças, agravos e desastres decorrentes das agressões ao meio ambiente, ao lado de novas estratégias de prevenção e controle de doenças com potencial de propagação internacional, colocadas pelo RSI (2005), apontam para a necessidade de readequação e aprimoramento de conceitos, estruturas, processos e práticas de vigilância em saúde, de forma articulada com a rede de atenção à saúde.

As evidências das mudanças climáticas globais e os possíveis efeitos na ocorrência de doenças (Intergovernmental..., 2007), como o aumento na magnitude e a ampliação da distribuição geográfica de doenças transmitidas por vetores e zoonoses, e a intensificação de desastres ambientais, também apontam para a reformulação das estratégias atualmente adotadas e reforçam a necessidade de articulação com outras áreas como meio ambiente, agricultura, defesa civil, entre outras.

No presente capítulo, buscou-se introduzir o novo conceito de emergência de saúde pública, que pode ser mais útil para a prática dos serviços de vigilância em saúde, na medida em que pode ser aplicado para situações em que o dano à saúde da população ainda não esteja estabelecido, permitindo a adoção de medidas oportunas que evitem ou reduzam a ocorrência de casos ou mortes. Ao ampliar o objeto de atuação da vigilância em saúde para além das doenças transmissíveis, para as quais esse conceito também pretende dar conta, será possível aumentar a capacidade dos serviços para atuar em um conjunto maior de problemas de saúde, que impliquem risco de disseminação de doenças. O exercício da utilização desse conceito pelos países, no âmbito do estabelecido pelo RSI (2005), e, no contexto nacional, pela Rede Cievs e por outras estruturas estaduais e municipais de serviços de saúde permitirá a sua consolidação, aperfeiçoamento e melhor aplicação na prática dos serviços.

A análise das emergências de saúde pública de importância nacional em nosso país tem permitido detectar algumas particularidades do padrão epidemiológico, bem como a diversidade existente entre regiões e Estados. Ainda que a comparação com outros países não seja plenamente possível, na medida em que as publicações internacionais têm destacado a distribuição de doenças infecciosas emergentes, é possível observar alguma similaridade com alguns desses estudos (Jones et al, 2008; Woolhouse, 2008), como a predominância de zoonoses e doenças de transmissão vetorial, também observada em nosso país. Por sua vez, outro grupo de eventos de saúde, os desastres ambientais, que em geral têm pouca visibilidade para os serviços de saúde e nas publicações internacio- 
nais, podem apresentar um impacto mais relevante na população, seja em número de pessoas diretamente afetadas, como pessoas desabrigadas ou acidentadas, seja por meio de efeitos secundários, como as doenças infecciosas ou traumas psicológicos decorrentes de enchentes, inundações e outros desastres.

As novas estratégias de enfrentamento das emergências de saúde pública vão requerer contínua avaliação dos seus resultados, com vistas a acompanhar as mudanças na dinâmica de transmissão e propagação de agentes e doenças, bem como adequá-las aos sistemas de saúde em todos os níveis de organização. Tendo como objetivo a estruturação desde o nível local, para que tenha capacidade de detectar, analisar, investigar e responder de forma rápida e eficiente a uma emergência de saúde pública, o sucesso dessa tarefa dependerá da adoção de medidas que sejam sustentáveis e favoreçam o fortalecimento do Sistema Único de Saúde.

Notas

l O Regulamento Sanitário Internacional anterior foi aprovado em 1969.

2 Estado Parte do RSI, ou seja, o Estado Membro da OMS para o qual o RSI entrou em vigor.

3 Estabelecido por meio da Portaria n.5 da Secretaria de Vigilância em Saúde, em 21.2.2006

Referências bibliográficas

BARRETO, M. L. et al. Infectious diseases epidemiology (Glossary). Journal of Epidemiology and Community Health, v.60, p.192-5, 2006.

CENTERS FOR DISEASE CONTROL AND PREVENTION. Addressing emerging infectious disease threats: a prevention strategy for the United States. Atlanta, Georgia: US Department of Health and Human Services, Public Health Services, 1994. 47p.

FIDLER, D. P.; GOSTIN, L. O. The new International Health Regulations: An historic development for international law and public health. Journal of Law, Medicine and Ethics, p.85-94, 2006.

INSTITUTE OF MEDICINE. Emerging infections: microbial threats to health in the United States. Washington, DC: National Academy Press, 1992.

. Microbial threats to health: emergency, detection and response. Washington, DC: National Academy Press, 2003. 367p.

INTERGOVERNMENTAL PANEL ON CLIMATE CHANGE. Climate change: synthesis report. Valencia, Spain, 2007. Disponível em: <http://www.ipcc.ch>.

JONES, K. E. et al. Global trends in emerging infectious diseases. Nature, v.451, p.9904, 2008. 
MINISTÉRIO DA SAÚDE. Secretaria de Vigilância em Saúde. Portaria n.5 da Secretaria de Vigilância em Saúde de 21.2.2006. Disponível em: <http://www.saude .gov. br/svs>.

WOOLHOUSE, M. E. J. Emerging diseases go global. Nature, v.451, p.898-9, 2008.

WORLD HEALTH ASSEMBLY, 2005. Revision of the International Health Regulations, WHA 58.3 (May 23, 2005) Disponível em: <http://www.who.int>.

RESUMO - Nos últimos anos, tem havido uma maior preocupação internacional com a disseminação ou propagação de agentes etiológicos e doenças, de natureza infecciosa, química e radionuclear. Visando adequar os conceitos e as medidas para evitar ou reduzir o risco dessa disseminação, foi adotado pelos países o novo conceito de emergência de saúde pública de importância internacional, no âmbito do RSI (2005), e implementadas novas estratégias para preparação e resposta a essas emergências. Com base em análise de risco contextualizada sobre eventos de saúde que podem se disseminar internacionalmente, busca-se contar com instrumentos mais oportunos que permitam a identificação e a atuação precoce sobre esses eventos. A adaptação desse conceito, para o propósito da vigilância em saúde e rede de atenção dos serviços de saúde nacional, tem permitido um melhor gerenciamento dos eventos de saúde que constituem risco de disseminação ou propagação de doenças ou agravos dentro do território nacional, além de oferecer uma resposta mais oportuna. A análise sobre esses eventos, realizada no presente trabalho, indica que a ocorrência de desastres ambientais, em número mais reduzido quando comparada com os eventos de natureza infecciosa, coloca em situação de risco a população ou gera danos à saúde em um contingente populacional maior. Por sua vez, os eventos de natureza infecciosa, que em sua maioria ocorrem sob a forma de surtos ou epidemias, acometem um maior número de municípios e apresentam maior letalidade. As medidas adotadas no país para aperfeiçoar o sistema de vigilância em saúde na detecção, preparação e resposta às emergências de saúde pública são descritas neste artigo e são destacados os principais desafios colocados pelos níveis de gestão do sistema.

PALAVRAS-CHAVE: Epidemias, Emergência de saúde pública, Doenças emergentes, Preparação e resposta em saúde pública.

ABSTRACT - During recent years has increased international concern about the spread of diseases or agents from infectious, chemical or radio-nuclear sources. Aiming to adapt the concepts and measures to prevent or reduce the risk of this spread, was adopted by the countries the new concept of "public health emergency of international concern" under the IHR (2005), and implemented new strategies for strengthening the activities to prepare and response to such emergencies. Based on contextualized risk analysis of health events that can spread internationally, one search to get more appropriate tools to timely identification and intervention on these events. The adaptation of this concept for the purpose of national health surveillance and health care services in Brazil, has allowed a better management of health events that may represent risk of spread of diseases or in- 
juries in the national territory and provide a more timely response. The analysis of these events, presented in this work, shows that the occurrence of environmental disasters with fewer number of events than infectious events, may constitute risk of damage or affect directly larger contingent of population. On the other hand, the infectious events, which usually occur as outbreaks or epidemics, affect a larger number of municipalities and have higher lethality. The measures adopted at the country to improve the health surveillance system on detection, preparedness and response to public health emergencies are described here and the main challenges in terms of managing this system are highlighted.

KErWORDS: Epidemics, Public health emergency, Emerging diseases, Public health preparedness and response.

Eduardo Hage Carmo é diretor de Vigilância Epidemiológica da Secretaria de Vigilância em Saúde, Ministério da Saúde. É médico, epidemiologista, doutor em Saúde Pública pelo Instituto de Saúde Coletiva da Universidade Federal da Bahia.

@ - eduardo.carmo@saude.gov.br

Gerson Penna é secretário de Vigilância em Saúde, Ministério da Saúde, médico dermatologista, doutor em Medicina Tropical, pesquisador adjunto IV do Núcleo de Medicina Tropical, Universidade de Brasília. @ - gpenna@saude.gov.br

Wanderson Kleber de Oliveira é coordenador do Centro de Informações Estratégicas e Respostas em Vigilância em Saúde, Secretaria de Vigilância em Saúde, Ministério da Saúde. É enfermeiro epidemiologista, mestrando em Epidemiologia pela UFRGS e especialista em Epidemiologia de Campo. @ - wanderson.kleber@saude.gov.br

Recebido em 14.10.2008 e aceito em 20.10.2008. 